\title{
Re-Entrant Quantum Phase Transitions in Antiferromagnetic Spin-1 Ladders
}

\author{
Shoji Yamamoto \\ Department of Physics, Okayama University, Tsushima, Okayama 700-8530, Japan \\ Tôru Sakai \\ Department of Electronics, Tokyo Metropolitan Institute of Technology, Hino, Tokyo 191-0065, Japan \\ Akihisa Koga \\ Department of Applied Physics, Osaka University, Suita, Osaka 565-0871, Japan
}

(Received 31 December 2001)

\begin{abstract}
In response to recent chemical attempts to construct higher-spin ladder materials from organic polyradicals, we study the ground-state properties of a wide class of antiferromagnetic spin-1 ladders. Employing various numerical tools, we reveal the rich phase diagram and correct a preceding nonlinear-sigma-model prediction. A variational analysis well interprets the phase competition with particular emphasis on the re-entrant phase boundary as a function of the rung interaction.
\end{abstract}

PACS numbers: 75.10.Jm, 75.40.Mg, 75.40.Cx

In 1983 Haldane [1] awoke renewed interest in quantum spin chains predicting a striking contrast between integer- and half-odd-integer-spin Heisenberg antiferromagnets. His argument was indeed verified in a spin-1 material $\mathrm{Ni}\left(\mathrm{C}_{2} \mathrm{H}_{8} \mathrm{~N}_{2}\right)_{2} \mathrm{NO}_{2}\left(\mathrm{ClO}_{4}\right)$ [2] and was given an analytic support [3] as well. Since then the energy gaps in magnetic excitation spectra, that is, spin gaps, have been a central issue in materials science. In the last decade more and more researchers made a wide variety of explorations into the spin-gap problem, such as the spinPeierls transition in inorganic compounds [4], quantized plateaux in magnetization curves [5], and antiferromagnetic gaps in the ferromagnetic background [6]. Among others Dagotto et al. [7] pointed out that another mechanism of the gap formation should lie in a ladder-two coupled chains. A spin gap was indeed observed in a typical two-leg ladder material $\mathrm{SrCu}_{2} \mathrm{O}_{3}$ [8]. Moreover, superconductivity was brought about in its hole-doped version $(\mathrm{SrCa}){ }_{14} \mathrm{Cu}_{24} \mathrm{O}_{41}$ [9]. Ladder systems caused us further surprise exhibiting excitation spectra varying with the number of their legs [10].

So far metal oxides have been representative of ladder materials. Though molecule-based ones [11,12 have been synthesized in an attempt to reduce the spin gaps and obtain experimental access to them, the situation of copper ions supplying the relevant spins remains unchanged. Therefore they are all spin- $\frac{1}{2}$ antiferromagnets. In such circumstances there has occurred a brand-new idea of constructing purely organic ladder systems. Katoh et al. 113 synthesized novel organic biradicals and tetraradicals which crystallize to form an antiferromagnetic ladder of spin- $\frac{1}{2}$ and that of effectively spin-1, respectively. Their polyradical strategy has yielded further harvest such as an effective spin-1 antiferromagnet on a honeycomb lattice 114 and a ladder ferrimagnet of mixed spins 1 and $\frac{1}{2}$ [15], displaying the wide tunability of the crystalline structures in higher-spin systems as well.

Distinct spin-gap mechanisms may lie in higher-spin ladders and quantum competition between them must lead us to further enthusiasm for ladder systems. There exist pioneering works in this context. Sierra [16] generalized the well-known nonlinear-sigma-model analysis [1,17] on quantum spin chains to multi-leg ladder systems. His findings suggest that only an odd number of half-odd-integer-spin chains are massless, supporting experimental observations on a series of spin- $\frac{1}{2}$ ladder antiferromagnets [8:18]. The technique was further developed for spatially inhomogeneous ladders 19. Mixedspin ladders [20 21 were also investigated with particular emphasis on the competition between massive and massless phases.

In comparison with extensive calculations [7, 22, 24 on spin- $\frac{1}{2}$ ladders, there exist few quantitative investigations into higher-spin ladders [25] and most of the above-mentioned predictive theories remain to be verified. Hence, in this article, we solve the ground-state properties of antiferromagnetic spin-1 ladders with two legs. Employing various numerical tools and complementing them by a variational argument, we elucidate the valence-bond-solid-like nature of their ground states, which is in contrast with the spin-liquid or resonatingvalence-bond ground states [22,23,26,27] of spin- $\frac{1}{2}$ twoleg ladders. The obtained phase diagram is reminiscent of the preceding sigma-model prediction [19] but contains a re-entrant phase boundary, which can never be extracted from any field-theoretical argument.

Considering that an advantage of assembling organic open-shell molecules into a magnetic material is the isotropic intermolecular exchange couplings, while the polyradical strategy is accompanied by spatial variations in magnetic interaction [14], we treat a wide class of spin1 antiferromagnetic ladders

$$
\mathcal{H}=\sum_{j=1}^{L}\left(\sum_{i=1}^{2} J_{\|} \gamma_{i, j} \boldsymbol{S}_{i, j} \cdot \boldsymbol{S}_{i, j+1}+J_{\perp} \boldsymbol{S}_{1, j} \cdot \boldsymbol{S}_{2, j}\right)
$$




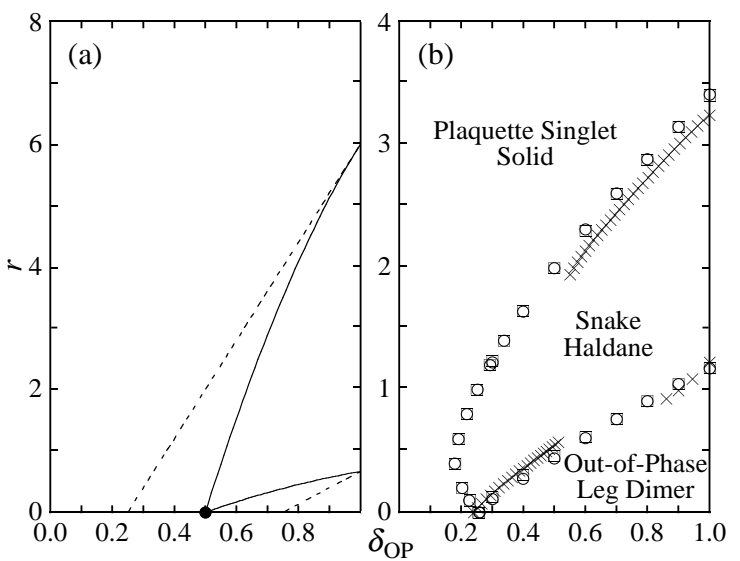

FIG. 1. Phase diagrams for the antiferromagnetic spin-1 ladder with two out-of-phase legs. (a) A field-theoretical prediction [19]. The two critical lines (dashed lines) derived from the effective sigma model for ladders are inconsistent with the sigma-model analysis on isolated chains $(\bullet)$. They remain far apart from each other even in the decoupled-chain limit $r=0$. Therefore qualitatively patched-up phase boundaries (solid lines) were predicted. (b) Our numerical findings. The series-expansion estimates are shown by $\times$, while the level-spectroscopy analyses by $\square(L=6)$ and $\circ(L=8)$.

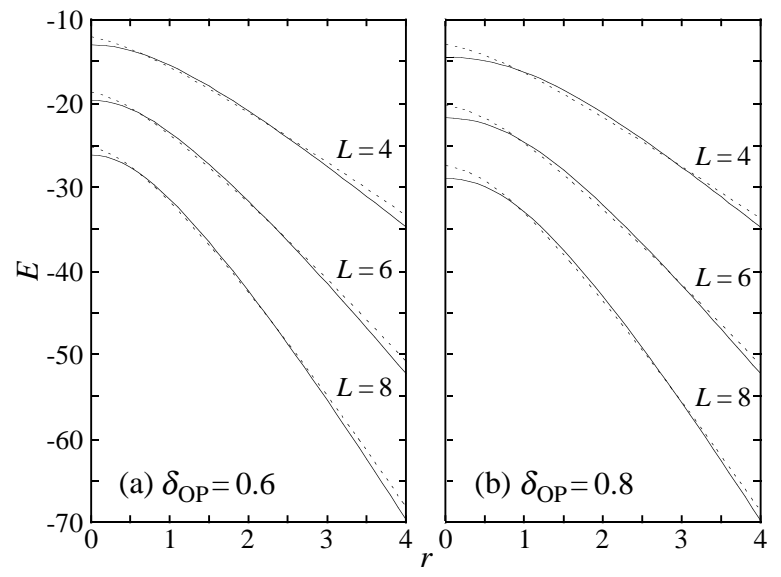

FIG. 2. Demonstration of the level spectroscopy. The lowest-lying two eigenvalues in the subspace of zero magnetization as functions of $r$ cross at transition points provided the twisted boundary condition is imposed on the Hamiltonian.

where the bond-alternation parameter $\gamma_{i, j}$ is defined in two ways as

$$
\gamma_{i, j}= \begin{cases}1+(-1)^{i+j} \delta_{\mathrm{OP}} & \text { (out-of-phase legs) }, \\ 1+(-1)^{j} \delta_{\mathrm{IP}} & \text { (in-phase legs). }\end{cases}
$$

We calculate the region of $0 \leq \delta_{\mathrm{OP}}\left(\delta_{\mathrm{IP}}\right) \leq 1$ and hereafter set $J_{\perp} / J_{\|}$to $r(\geq 0)$. Martin-Delgado, Shankar, and Sierra 19] studied the cases of out-of-phase legs deriving a low-energy-relevant sigma model. For the spin- $S$ ladders with two out-of-phase legs, the topological angle in the effective sigma model turns out $8 \pi S \delta_{\mathrm{OP}} /(r+2)$ and reads as the critical lines $8 S \delta_{\mathrm{OP}}=(2 n+1)(r+2)$ $(n=0, \pm 1, \cdots)$. However, these findings do not smoothly merge with the well-established critical behavior in one dimension, $2 S(1-\delta)=2 n+1$ [17], as is shown in Fig. 1(a). Thus, it is necessary to verify the true scenario all the more in higher dimensions.

One of the most reliable solution may be a numerical analysis 28 on the phenomenological renormalizationgroup equation [29]. However, the scaled gaps are illnatured due to the close critical points, so as to make the fixed points hard to extract from available numerical data. Then we switch our strategy to the level spectroscopy [30], the core idea of which is summarized as detecting transition points by crossing of two relevant energy levels. Although the method is generically applicable to the Gaussian critical points [31], no explicit change of symmetry accompanies the present phase transitions and therefore any levels do not cross naively. In order to overcome the difficulty of this kind, Kitazawa 32] proposed the idea of applying the twisted boundary condition, that is to say in the present case, setting the boundary exchange couplings equal to $-\sum_{i=1}^{2} J_{\|} \gamma_{i, L}\left(S_{i, L}^{x} S_{i, 1}^{x}+S_{i, L}^{y} S_{i, 1}^{y}-S_{i, L}^{z} S_{i, 1}^{z}\right)$. Then the energy structure of the Hamiltonian is changed and the lowest two levels are led to cross at transition points, which is demonstrated in Fig. 2. Due to the limit of time and memory well spent, we have restricted our calculations up to $L=8$. We plot in Fig. 11(b) bare findings for the crossing points at $L=6$ and $L=8$ rather than extrapolate them trickily. We are sure that the data uncertainty still left is within the symbol size. A series-expansion technique 33, 34] guarantees the level spectroscopy to work well. Starting with decoupled singlet dimers on legs or rungs and expanding the energy gap as a power series in a relevant perturbation parameter, we can obtain a partial knowledge of phase transitions. Here we have calculated the gap up to the ninth order and further applied the Dlog Padé approximants [35] to them. The thus-obtained phase boundaries, which are also shown in Fig. 1 (b), elucidate the nature of the phase competition, that is, the Affleck-Kennedy-Lieb-Tasaki (AKLT) valence-bond-solid (VBS) [3] on a snakelike path versus decoupled dimers.

The most impressive findings are re-entrant quantum phase transitions with increasing $r$. The preceding sigmamodel analysis [19] is indeed enlightening but never able to reveal this novel quantum behavior. In order to characterize each phase, let us consider a variational approach. We know that singlet dimers on rungs [Fig. 3(h)] are stabilized for $r \rightarrow \infty$, whereas either dimers on legs [Figs. 3(d) and 3(e)] or the double AKLT VBS [Fig. 3(c)] for $r \rightarrow 0$. Two more interchain VBS states [Figs. B(f) and $3(\mathrm{~g})]$ may be adopted as variational components for the intermediate- $r$ region. Thus the linear combination of Figs. 3(c) to $3(\mathrm{~h})$ can be an approximate ground-state wave function for spin-1 ladders. Since the present variational components are all asymptotically orthogonal to each other, the variational ground state turns out any of them itself [36]. The thus-obtained phase diagram is 
presented in Fig. 4. The significant stabilization of the intermediate phase, which is now characterized as $\mathrm{SH}$, and the resultant re-entrant phase boundary are successfully reproduced. Considering that a couple of critical chains immediately turn massive with their rung interaction switched on [7], the point $\mathrm{C}$ should coincide with the point A under more refined (and thus inevitably numerical) variational investigation.

The present variational calculation implies possible phase transitions for in-phase-leg ladders as well, but this is totally due to the naive wave function. Numerical observation of the energy structure ends up with no gapless

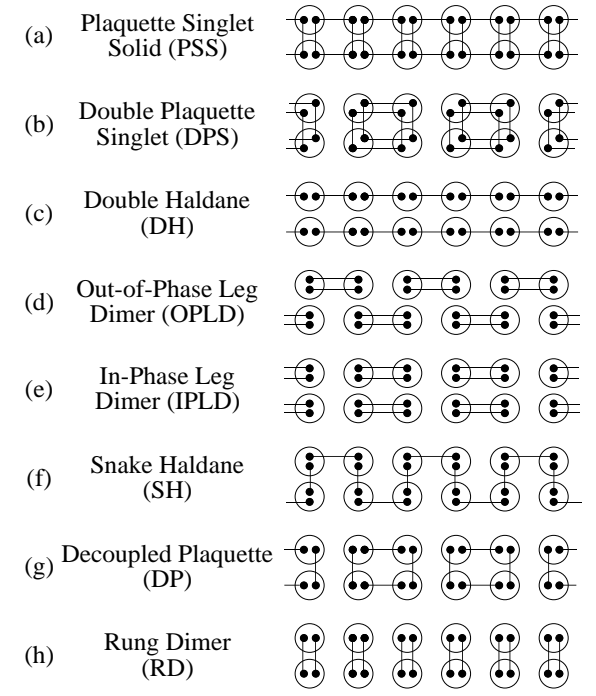

FIG. 3. Plaquette-singlet-solid and valence-bond-solid states relevant to the two-leg antiferromagnetic spin-1 ladders. - denotes a spin $\frac{1}{2}$ and their segment linkage means a singlet formation. $\bigcirc$ represents an operation of constructing a spin 1 by symmetrizing the two spin $\frac{1}{2}$ 's inside.

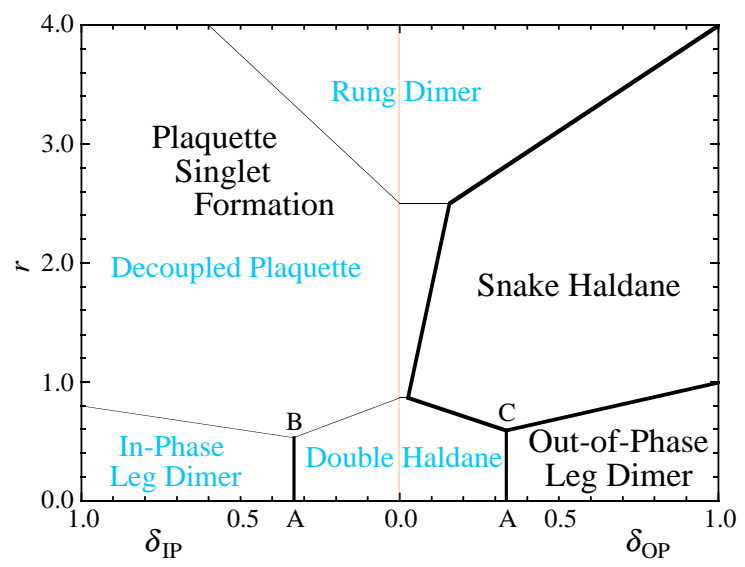

FIG. 4. Variational phase diagrams for the two-leg antiferromagnetic spin-1 ladders. The thick solid lines describe phase transitions, whereas the thin ones represent the crossover of the ground-state nature within the present variational scheme. The dotted line is only a guide for eyes. point in this region. The sigma-model approach also concludes no critical point, giving the topological angle $4 \pi S$ independent of both $r$ and $\delta_{\mathrm{P}}$. The key to the ground-state nature of in-phase-leg ladders is the fourspin correlation 25. Let us consider interacting four spins of $S=\frac{1}{2}$ which are described by the Hamiltonian $\mathcal{H}=\sum_{i=1}^{2} \boldsymbol{S}_{i, l} \cdot \boldsymbol{S}_{i, l+1}+r \sum_{j=l}^{l+1} \boldsymbol{S}_{1, j} \cdot \boldsymbol{S}_{2, j}$. In terms of the Schwinger boson representation: $S^{+}=a^{\dagger} b ; S^{-}=a b^{\dagger}$; $S^{z}=\frac{1}{2}\left(a^{\dagger} a-b^{\dagger} b\right) ; \hat{S}=\frac{1}{2}\left(a^{\dagger} a+b^{\dagger} b\right)$, their ground state is explicitly given as

$$
\begin{array}{r}
|\psi(r)\rangle_{l}=\left[\cos \theta(r) \prod_{i=1}^{2}\left(a_{i, l}^{\dagger} b_{i, l+1}^{\dagger}-b_{i, l}^{\dagger} a_{i, l+1}^{\dagger}\right)\right. \\
\left.+\sin \theta(r) \prod_{j=l}^{l+1}\left(a_{1, j}^{\dagger} b_{2, j}^{\dagger}-b_{1, j}^{\dagger} a_{2, j}^{\dagger}\right)\right]|0\rangle_{l}
\end{array}
$$

where $|0\rangle_{l}$ is the Bose vacuum and $\theta(r)$ is given by $\tan \theta(r)=r-1+\sqrt{1-r+r^{2}}$. As $r$ varies from 0 to $\infty$, $\theta$ moves from 0 to $\frac{\pi}{2}$, that is, the leg dimers continuously turn into the rung dimers. Using the plaquette singlet state (3), we can in principle construct much better variational wave functions for spin-1 ladders particularly in the in-phase-leg region as

$$
\begin{aligned}
& |\Psi(r)\rangle=\prod_{i, j} \mathcal{P}_{i, j} \prod_{l}|\psi(r)\rangle_{2 l-1} \\
& \quad \times\left[\cos \phi\left(\delta_{\mathrm{IP}}\right)|\psi(r)\rangle_{2 l-1}+\sin \phi\left(\delta_{\mathrm{IP}}\right)|\psi(r)\rangle_{2 l}\right],
\end{aligned}
$$

where $\mathcal{P}_{i, j}$ represents an operation of symmetrizing the two spin $\frac{1}{2}$ 's at site $(i, j)$ into a spin 1 . Now that $\theta(r)$ is a continuous function of $r$ and may here deviate from that in Eq. (3), a naive optimization [36] of Eq. (4) is no more feasible. However, the refined variational scheme shows us more. For better understanding of the wave function (4), we visualize in Fig. 3 its special forms for $\phi=\frac{\pi}{2}$ (a) and $\phi=0$ (b), which are most stabilized at $\delta_{\mathrm{IP}}=0$ and $\delta_{\mathrm{IP}}=1$, respectively. The snapshots of PSS (DSP) at $\theta=0, \theta=\frac{\pi}{4}$, and $\theta=\frac{\pi}{2}$ are nothing but the variational components $\mathrm{DH}$ (IPLD), DP, and RD, respectively. Through the plaquette singlet resonance, any snapshot of PSS (DPS) can turn into another without any explicit transition. PSS and DPS share DP and $\mathrm{RD}$ as their snapshots. Thus, the extended variational wave function (4) is expected to erase the artificial firstorder transition lines (thin solid lines), reducing the only discontinuity wall AB to a point. The point A belongs to the same universality class as the spin- $\frac{1}{2}$ Heisenberg chain 31]. We are all the more convinced of the immediate gap formation with $r$ moving away from 0 . On the other hand, neither PSS nor DPS includes both OPLD and $\mathrm{SH}$ and therefore the two critical lines in the outof-phase-leg region survive against the plaquette singlet formation.

The generalized string order parameter 37] $O(\theta)=$ $\lim _{|i-j| \rightarrow \infty}\left\langle S_{i}^{z} \prod_{l=i}^{j-1} \exp \left[\mathrm{i} \theta S_{l}^{z}\right] S_{j}^{z}\right\rangle$ is also useful in char- 
acterizing the ground state. $O(\theta)$ distinguishes between one-dimensional VBS states by its $\theta$ dependence [36. Hence, measuring it on the linear-chain and snake paths, we can detect the transitions between DH, OPLD, SH, and $\mathrm{RD}$, as is shown in Fig. 5. If we specify the transition through a change of the $\theta$ dependence in the vicinity of $\theta=\pi$, that is, the change from the convex curve to the concave one, we obtain the transition points $\left(\delta_{\mathrm{OP}}, r\right)=(0.245,0)$ and $(0.6,2.125)$, which are in good agreement with the numerical findings in Fig. 1(b). Whatever path we take for $O(\theta)$, its peak never sits on $\theta=\pi$ in the in-phase-leg region, suggesting that we can not observe the Haldane state of any kind there. The plaquette singlet formation can instead be visualized by extending $O(\theta)$ to ladders as $\lim _{|i-j| \rightarrow \infty}\left\langle S_{1, i}^{z} S_{2, i}^{z} \prod_{l=i}^{j-1} \exp \left[\mathrm{i} \theta\left(S_{1, l}^{z}+S_{2, l}^{z}\right)\right] S_{1, j}^{z} S_{2, j}^{z}\right\rangle[25$.

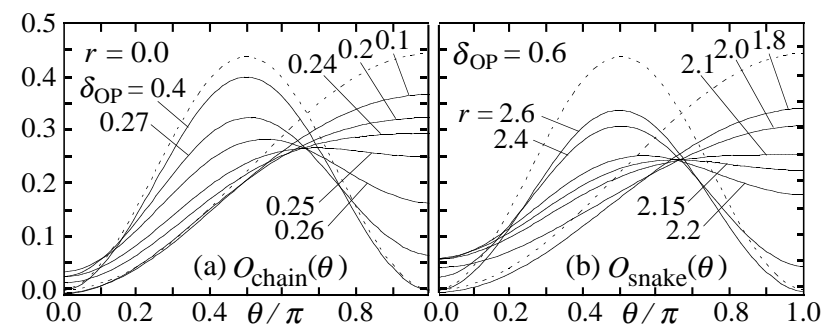

FIG. 5. Quantum Monte Carlo estimates of the generalized string order parameter defined on the linear-chain (a) and snake (b) paths. The dashed lines represent the analytic calculations for the AKLT VBS $\left(\frac{4}{9} \sin ^{2} \frac{\theta}{2}\right)$ and decoupled dimers $\left(\frac{4}{9} \sin ^{2} \theta\right) . \delta_{\text {OP }}$ runs on the line of $r=0.0$ in (a), while $r$ runs on the line of $\delta_{\mathrm{OP}}=0.6$ in (b).

We summarize our rich harvest as the plaquette singlet formation and the re-entrant quantum phase transition via the snake Haldane state in staggered spin-1 ladders. The re-entrant phase boundary is peculiar to spin-1 or possibly integer-spin ladders. Half-odd-integerspin chains are generically critical at the translationally symmetric point $\delta=0$. Therefore, the critical line can not exhibit the initial re-entrant behavior in the $r-\delta$ phase diagram, because it should be symmetric for $\pm \delta$. Chemical tuning of bond-alternating critical chain compounds [38], as well as the organic-radical-based materials research [13], is strongly encouraged.

We are grateful to H. Takayama and K. Okamoto for fruitful discussions. This work was supported by the Japanese Ministry of Education, Science, Sports, and Culture. The numerical calculation was done using the facility of the Supercomputer Center, Institute for Solid State Physics, University of Tokyo.

[1] F. D. M. Haldane: Phys. Lett. 93A (1983) 464; Phys.
Rev. Lett. 50 (1983) 1153.

[2] J. P. Renard, M. Verdaguer, L. P. Regnault, W. A. C. Erkelens, J. Rossat-Mignod and W. G. Stirling: Europhys. Lett. 3 (1987) 945.

[3] I. Affleck, T. Kennedy, E. H. Lieb and H. Tasaki: Phys. Rev. Lett. 59 (1987) 799; Commun. Math. Phys. 115 (1988) 477.

[4] M. Hase, I. Terasaki and K. Uchinokura: Phys. Rev. Lett. 70 (1993) 3651.

[5] M. Oshikawa, M. Yamanaka and I. Affleck: Phys. Rev. Lett. 78 (1997) 1984.

[6] S. Yamamoto and T. Fukui: Phys. Rev. B 57 (1998) R14008.

[7] E. Dagotto, J. Riera and D. Scalapino: Phys. Rev. B 45 (1992) R5744.

[8] M. Azuma, Z. Hiroi, M. Takano, K. Ishida and Y. Kitaoka: Phys. Rev. Lett. 73 (1994) 3463.

[9] M. Uehara, T. Nagata, J. Akimitsu, H. Takahashi, N. Môri and K. Kinoshita: J. Phys. Soc. Jpn. 65 (1996) 2764 .

[10] E. Dagotto and T. M. Rice: Science 271 (1996) 618.

[11] C. A. Hayward, D. Poilblanc and L. P. Lévy: Phys. Rev. B 54 (1996) R12649.

[12] C. P. Landee, M. M. Turnbull, C. Galeriu, J. Giantsidis and F. M. Woodward: Phys. Rev. B 63 (2001) R100402.

[13] K. Katoh, Y. Hosokoshi, K. Inoue and T. Goto: J. Phys. Soc. Jpn. 69 (2000) 1008.

[14] Y. Hosokoshi, Y. Nakazawa, K. Inoue, K. Takizawa, H. Nakano, M. Takahashi and T. Goto: Phys. Rev. B 60 (1999) 12924.

[15] Y. Hosokoshi, K. Katoh, Y. Nakazawa, H. Nakano and K. Inoue: to be published in J. Am. Chem. Soc..

[16] G. Sierra: J. Phys. A: Math. Gen. 29 (1996) 3299.

[17] I. Affleck: Nucl. Phys. B257 (1985) 397; B265 (1986) 409.

[18] R. S. Eccleston, T. Barnes, J. Brody and J. W. Johnson: Phys. Rev. Lett. 73 (1994) 2626.

[19] M. A. Martin-Delgado, R. Shankar and G. Sierra: Phys. Rev. Lett. 77 (1996) 3443.

[20] T. Fukui and N. Kawakami: Phys. Rev. B 57 (1998) 398.

[21] A. Langari, M. Abolfath and M. A. Martin-Delgado: Phys. Rev. B 61 (2000) 343.

[22] A. Parola and S. Sorella and Q. F. Zhong: Phys. Rev. Lett. 71 (1993) 4393.

[23] S. R. White, R. M. Noack and D. J. Scalapino: Phys. Rev. Lett. 73 (1994) 886.

[24] B. Frischmuth, B. Ammon and M. Troyer: Phys. Rev. B 54 (1996) R3714.

[25] S. Todo, M. Matsumoto, C. Yasuda and H. Takayama: cond-mat/0107115.

[26] P. W. Anderson: Science 235 (1987) 1196.

[27] T. M. Rice, S. Gopalan and M. Sigrist: Europhys. Lett. 23 (1993) 445.

[28] T. Sakai and S. Yamamoto: Phys. Rev. B 60 (1999) 4053.

[29] M. P. Nightingale: Physica A 83 (1976) 561.

[30] K. Nomura and K. Okamoto: J. Phys. A: Math. Gen. 27 (1994) 5773.

[31] S. Yamamoto: Phys. Rev. B 51 (1995) 16128; 52 (1995) 10170.

[32] A. Kitazawa: J. Phys. A: Math. Gen. 30 (1997) L285. 
[33] R. R. P. Singh, M. P. Gelfand and D. A. Huse: Phys. Rev. Lett. 61 (1988) 2484.

[34] A. Koga and N. Kawakami: Phys. Rev. 61 (2000) 6133.

[35] A. J. Guttmann: Phase Transitions and Critical Phenomena, ed. C. Domb and J. L. Lebowitz (Academic,
New York, 1989) Vol. 13.

[36] S. Yamamoto: Phys. Rev. B 55 (1997) 3603.

[37] M. Oshikawa: J. Phys.: Condens. Matter 4 (1992) 7469.

[38] M. Hagiwara, Y. Narumi, K. Kindo, H. Nakano, R. Sato and M. Takahashi: Phys. Rev. Lett. 80 (1998) 1312. 